\title{
Revealed preference analysis for convex rationalizations on nonlinear budget sets
}

\author{
Laurens Cherchye* Thomas Demuynck ${ }^{\dagger} \quad$ Bram De Rock $^{\ddagger}$
}

April 29, 2014

\begin{abstract}
We present necessary and sufficient revealed preference conditions to verify whether a finite data set on nonlinear budget sets is consistent with the maximization of a quasi-concave utility function. When budget sets are finite unions of polyhedral convex sets, these conditions require feasibility of a set of linear inequalities, which makes them easy to use in practical applications.

Keywords: quasi-concavity; convex preferences; nonlinear budget sets; revealed preference conditions
\end{abstract}

JEL: C14, D11

\section{Introduction}

Quasi-concavity of the utility function or, equivalently, convexity of the preference relation, corresponds to diminishing marginal rates of substitution: in order to keep utility constant, a consumer is willing to sacrifice less and less of a certain good for fixed increments of another good. Convex preferences are crucial to define shadow prices, which are often fundamental to the analysis of public goods and externalities in theories of optimal taxation, models of risk and ambiguity aversion, ... When preferences are non-convex, certain consumption bundles will not have a supporting price vector. This in turn can lead to non-convex valued demand correspondences, and might even lead to a failure of the second fundamental theorem of welfare economics, as it is possible that a Pareto optimal allocation is not supported by a set of equilibrium prices.

\footnotetext{
${ }^{*}$ Center for Economic Studies, University of Leuven, E. Sabbelaan 53, B-8500 Kortrijk, Belgium. Laurens Cherchye gratefully acknowledges the European Research Council (ERC) for his consolidator grant and the Research Fund K.U.Leuven for the grant STRT1/08/004. Email: Laurens.Cherchye@kuleuven.be

${ }^{\dagger}$ Maastricht University, Tongersestraat 53, 6370 Maastricht, Netherlands. Email: t.demuynck@maastrichtuniversity.nl

${ }^{\ddagger}$ ECARES, Université de Bruxelles; Avenue F.D. Roosevelt 50, CP 114, B-1050 Brussels, Belgium. Bram De Rock gratefully acknowledges the European Research Council (ERC) for his Starting Grant. Email: bderock@ulb.ac.be
} 
Given this revealed importance of convex preferences in the literature, one may have expected much attention for the testable implications of this assumption. However, the empirical demand literature is surprisingly silent on the issue. A first important explanation may be Afriat (1967)'s theorem. One of the most surprising implications of this theorem is that any finite data set on linear budgets is consistent with the maximization of a locally non-satiated utility function if and only if it is consistent with the maximization of an increasing and concave utility function. As such, it is impossible to reject quasi-concavity without rejecting the assumption of utility maximization. Essentially, this means that convexity of preferences is nontestable in the case of linear budgets. A second main explanation is due to the advent of duality theory. Currently, most empirical demand analysis starts from a specification of a cost function defined for linear budget sets, which exists even if the underlying utility function is not quasi-concave. In this manner, quasi-concavity is no longer necessary to make individual demand analysis empirically applicable, at least when budget sets are assumed to be linear.

Importantly, the above cited results only suggest that tests of convexity will be empirically idle in the case of linear budget sets. However, many economic decision situations are characterized by nonlinear budget sets. For example, nonlinear budgets prevail in labor supply settings where differentiated tax systems imply a nonlinear trade-off between leisure and consumption, in intertemporal consumption where different interest rates for borrowing and saving make a nonlinear exchange between current and future consumption, in game theoretic settings where individuals' behavior is mutually interdependent, and in models of household production with nonlinear technologies. ${ }^{1}$ These examples directly motivate our research question, i.e. characterize the testable implications of convex rationalizations on nonlinear budget sets.

This note provides an account of the revealed preference implications associated with convex preferences. We do so by considering budget sets that are possibly nonlinear. Our main insight is based on the concept of co-convex hulls, which figure as (approximations of) lower contour sets under convex rationalizations of a given data set. We will demonstrate that convex preferences do have testable implications as soon as budget sets are nonlinear. We build further on this theoretical result to come up with testable conditions of convex preferences that are easy to implement in practice. To this end, we consider the class of (possibly nonlinear) budget sets that can be defined as a finite union of polyhedral convex sets. While -admittedly- this simplifying assumption implies a somewhat narrow focus, it is actually hardly restrictive from an applied point of view. In many practical applications nonlinear budget sets fall within this class and if not, it is always possible to approximate any nonlinear budget set arbitrarily close as a finite union of polyhedral convex sets.

Our paper fits in the literature on revealed preference tests for consumption behavior to be rationalizable by utility maximization under nonlinear budgets. The paper in this literature which is closest to ours is probably Forges and Minelli (2009). Their main

\footnotetext{
${ }^{1}$ See Forges and Minelli (2009) for examples of game theoretic settings involving nonlinear budgets, and Deaton and Muellbauer (1980) for other examples of decision situations characterized by nonlinear budgets.
} 
result is presented in the next section. A second paper by Matzkin (1991) considers two extensions of Afriat's theorem to nonlinear budget sets. The first extension assumes that every budget set is co-convex. In this case she shows that the usual Afriat theorem still holds. The second extension assumes that every budget set has a unique supporting hyperplane through the chosen bundle that contains the whole budget. Matzkin demonstrated that, if we replace the budget set by the half space defined by this hyperplane, the data set is rationalizable by a concave utility function if and only if the usual revealed preference conditions are satisfied for these new 'virtual' (linear) budget sets. As will be clear from our discussion below, both extensions are special cases of our main result. Finally, our research question is also closely related to an original contribution of Yatchew (1985). This author also considered the case of rationalizability by a concave utility function when the budget set can be written as a finite union of polyhedral convex sets. He obtains a set of (quadratic) inequalities that are necessary and sufficient for consistency with a concave utility function. The differences between his and our results are discussed in more depth in Section 3.

The remainder of this paper is organized as follows. Section 2 sets the stage by introducing Afriat's theorem (for linear budget sets) and the rationalizability result of Forges and Minelli (2009) (for nonlinear budget sets). Here, we show that Afriat's equivalence result breaks down in the case of nonlinear budgets. We also present our main theoretical result, i.e. the analogue of Afriat's theorem for nonlinear budget sets. Section 3 tackles our empirical research question and considers the practical operationalization of our testable conditions for convex rationalizations. This section also illustrates the practical usefulness of our results for labor supply analysis.

\section{Afriat's theorem and testability of convexity}

In this section, we first introduce some useful notation and definitions. Next, we present Afriat's theorem. As indicated above, a main implication of this result is that convexity of preferences (or quasi-concavity of a rationalizing utility function) is nontestable under linear budget sets. Subsequently, we discuss the main theorem of Forges and Minelli (2009) and show that Afriat's nontestability result does not extend to nonlinear budget sets. Finally, we present a generalization of Afriat's theorem that characterizes the data sets with nonlinear budgets that are rationalizable by convex preferences, i.e. there exists a rationalization by a quasi-concave utility function.

Notation and definitions. A data set $S=\left\{B_{t}, \mathbf{x}_{t}\right\}_{t \in T}$ consists of a finite collection of subsets $B_{t}$ of $\mathbb{R}_{+}^{n}$ and elements $\mathbf{x}_{t} \in B_{t}$. The intuition is that $B_{t}$ is a budget set, which contains all feasible consumption bundles of $n$ goods at observation $t \in T$, while $\mathbf{x}_{t}$ is the chosen consumption bundle from this set. We call $\left(B_{t}, \mathbf{x}_{t}\right)$ an observation.

We impose three assumptions on any budget set $B_{t}$. First of all, we require that $B_{t}$ is closed. In other words, all limits of sequences of bundles in $B_{t}$ are also in $B_{t}$. This is a technical but generally uncontroversial assumption. Second, we assume that the sets $B_{t}$ are monotone. Formally, this implies for all $\mathbf{x} \in B_{t}$ and all $\mathbf{y} \in \mathbb{R}_{+}^{n}$, if $\mathbf{y} \leq \mathbf{x}$, 
then $\mathbf{y} \in B_{t}$. Intuitively, this condition states that, when an individual can afford the bundle $\mathbf{x}$, then she can also afford any bundle $\mathbf{y} \leq \mathbf{x} .{ }^{2}$ This assumption is satisfied if we assume that an individual can costlessly dispose of any amount of goods, i.e. $\mathbf{y}$ can be obtained by choosing $\mathbf{x}$ and throwing away the bundle $\mathbf{x}-\mathbf{y}$. Third, we assume that the set $B_{t}$ has a non-empty interior. This excludes settings where the budget set imposes zero consumption on a subset of goods. As a final note, we want to stress that we do not require our budget sets to be compact. As such, it is possible that budget sets are unbounded in some direction.

A bundle $\mathbf{x} \in B_{t}$ is on the boundary $\partial B_{t}$ of $B_{t}$ if there is no other bundle $\mathbf{y}$ in $B_{t}$ that contains more of every good than the bundle $\mathbf{x}$. Formally, $\partial B_{t}=\left\{\mathbf{x} \in B_{t} \mid \forall \mathbf{y} \gg\right.$ $\left.\mathbf{x}: \mathbf{y} \notin B_{t}\right\}$.

A budget set $B_{t}$ is linear if there exists a price vector $\mathbf{p}_{t} \in \mathbb{R}_{++}^{n}$ and a budget $m_{t} \in \mathbb{R}_{+}$ such that $B_{t}$ contains all bundles $\mathbf{x}$ of which the expenditure at prices $\mathbf{p}_{t}$ does not exceed $m_{t}$, i.e. $B_{t}=\left\{\mathbf{x} \in \mathbb{R}_{+}^{n} \mid \mathbf{p}_{t} \mathbf{x} \leq m_{t}\right\}$. Observe that, for linear budget sets, the boundary of $B_{t}$ coincides with the budget hyperplane; $\partial B_{t}=\left\{\mathbf{x} \in \mathbb{R}_{+}^{n} \mid \mathbf{p}_{t} \mathbf{x}=m_{t}\right\}$. If all budgets are linear and $m_{t}=\mathbf{p}_{t} \mathbf{x}_{t}$ for all $t \in T$, we also denote such data set by $\left\{\mathbf{p}_{t}, \mathbf{x}_{t}\right\}_{t \in T}$. This is the type of data set Afriat (1967) considered in his original study.

A utility function $u: \mathbb{R}_{+}^{n} \rightarrow \mathbb{R}$ associates with any conceivable bundle $\mathbf{x} \in \mathbb{R}_{+}^{n}$ a real number $u(\mathbf{x})$. We will consider the following properties of utility functions. A utility function $u$ is concave if, for all $\mathbf{x}, \mathbf{y} \in \mathbb{R}_{+}^{n}$ and $\alpha \in[0,1], u(\alpha \mathbf{x}+(1-\alpha) \mathbf{y}) \geq \alpha u(\mathbf{x})+(1-$ $\alpha) u(\mathbf{y})$. A utility function is quasi-concave if the better-than sets are convex. Formally, for all $\mathbf{x}, \mathbf{y}$ and $\mathbf{z} \in \mathbb{R}_{+}^{n}$ and $\alpha \in[0,1]$, we have that $u(\mathbf{x}) \leq \min \{u(\mathbf{y}), u(\mathbf{z})\} \Rightarrow u(\mathbf{x}) \leq$ $u(\alpha \mathbf{y}+(1-\alpha) \mathbf{z})$. A function $u$ is locally non-satiated if, for all bundles $\mathbf{x} \in \mathbb{R}_{+}^{n}$, there exists a bundle arbitrarily close to $\mathbf{x}$ that has higher utility than $\mathbf{x}$. Formally, for all open neighborhoods $N$ of $\mathbf{x}$, there exists a bundle $\mathbf{y}$ in $N \cap \mathbb{R}_{+}^{n}$ such that $u(\mathbf{y})>u(\mathbf{x})$. We say that a function $u$ is increasing if, for all $\mathbf{x}, \mathbf{y} \in \mathbb{R}_{+}^{n}, \mathbf{x} \gg \mathbf{y}$ implies $u(\mathbf{x})>u(\mathbf{y})$. Finally, a function $u$ is continuous if, for all $\mathbf{x} \in \mathbb{R}_{+}^{n}$, the upper contour sets $\left\{\mathbf{y} \in \mathbb{R}_{+}^{n} \mid u(\mathbf{y})>u(\mathbf{x})\right\}$ and the lower contour sets $\left\{\mathbf{y} \in \mathbb{R}_{+}^{n} \mid u(\mathbf{y})<u(\mathbf{x})\right\}$ are open subsets of $\mathbb{R}_{+}^{n}$. Of course, if $u$ is increasing and continuous, then we also have that $\mathbf{x} \geq \mathbf{y}$ implies $u(\mathbf{x}) \geq u(\mathbf{y})$.

A data set $S=\left\{B_{t}, \mathbf{x}_{t}\right\}_{t \in T}$ is said to be rationalizable if there exists a utility function that makes the observations consistent with utility maximization.

Definition 1 (Rationalizability). A data set $S=\left\{B_{t}, \mathbf{x}_{t}\right\}_{t \in T}$ is rationalized by the utility function $u: \mathbb{R}_{+}^{n} \rightarrow \mathbb{R}$ if, for all $t \in T, \mathbf{x}_{t}$ maximizes $u(\mathbf{x})$ subject to the condition $\mathbf{x} \in B_{t}$, i.e.

$$
\mathbf{x}_{t} \in \arg \max _{\mathbf{x} \in B_{t}} u(\mathbf{x})
$$

Afriat's theorem: linear budget sets. Building on the work of Afriat (1967), Varian (1982) presents a combinatorial condition for rationalizability by a concave, increasing and continuous utility function when all budget sets $B_{t}$ are linear. Specifically, the

\footnotetext{
${ }^{2}$ For two elements $\mathbf{x}, \mathbf{y} \in \mathbb{R}_{+}^{n}$ we have that $\mathbf{x} \leq \mathbf{y}$ if $x_{i} \leq y_{i}$ for each good $i \leq n$, we have that $\mathbf{x}<\mathbf{y}$ if $\mathbf{x} \leq \mathbf{y}$ and $\mathbf{x} \neq \mathbf{y}$ and we write $\mathbf{x} \ll \mathbf{y}$ if $x_{i}<y_{i}$ for all goods $i$.
} 
condition requires data consistency with the so-called generalized axiom of revealed preference or GARP. Given our specific focus, it is useful to formulate this GARP condition for a setting with general (possibly nonlinear) budget sets.

First we define the direct revealed preference relation $R_{0}$ as $\mathbf{x}_{t} R_{0} \mathbf{x}_{v}$ if $\mathbf{x}_{v} \in B_{t}$. In other words, the bundle $\mathbf{x}_{t}$ is directly revealed preferred to $\mathbf{x}_{v}$ if $\mathbf{x}_{t}$ was chosen but $\mathbf{x}_{v}$ was also available. The indirect revealed preference relation $R$ is the transitive closure of the direct revealed preference relation: $\mathbf{x}_{t} R \mathbf{x}_{v}$ if there exist a sequence $\mathbf{x}_{t}, \mathbf{x}_{k}, \mathbf{x}_{r}, \ldots, \mathbf{x}_{m}, \mathbf{x}_{v}$ such that $\mathbf{x}_{t} R_{0} \mathbf{x}_{k} ; \mathbf{x}_{k} R_{0} \mathbf{x}_{r}, \ldots$, and $\mathbf{x}_{m} R_{0} \mathbf{x}_{v}$. Finally, the data set $S=\left\{B_{t}, \mathbf{x}_{t}\right\}_{t \in T}$ is said to satisfy GARP if for all $\mathbf{x}_{t}$ and $\mathbf{x}_{v}$, if $\mathbf{x}_{t} R \mathbf{x}_{v}$, then it is not the case that $\mathbf{x}_{t} \in B_{v} \backslash \partial B_{v}$. In words, if $\mathbf{x}_{t}$ is (indirectly) revealed preferred to $\mathbf{x}_{v}$, then $\mathbf{x}_{t}$ should not be in the interior of $B_{v}$. In the case of linear budget sets, this requires $\mathbf{p}_{v} \mathbf{x}_{v} \leq \mathbf{p}_{v} \mathbf{x}_{t}$ whenever $\mathbf{x}_{t} R \mathbf{x}_{v}$. Observe that for every chosen bundle $\mathbf{x}_{t}$ we automatically have that $\mathbf{x}_{t} R \mathbf{x}_{t}$. As such, GARP requires that each chosen bundle $\mathbf{x}_{t}$ must be on the boundary of its own budget set $B_{t}$, i.e. $\mathbf{x}_{t} \in \partial B_{t}$.

We are now in a position to state Afriat's theorem (Varian (1982), based on Afriat (1967)). This result characterizes rationalizable data sets $S$ in the case of linear budget sets.

Theorem 1 (Afriat's theorem). Consider a data set $S=\left\{\mathbf{p}_{t}, \mathbf{x}_{t}\right\}_{t \in T}$ with linear budget sets. Then, the following statements are equivalent:

(i) The data set $S$ is rationalizable by a locally non-satiated utility function.

(ii) The data set $S$ satisfies GARP.

(iii) For all $t \in T$, there exist numbers $U_{t} \geq 0$ and $\lambda_{t}>0$ such that, for all $t, v \in T$ :

$$
U_{t}-U_{v} \leq \lambda_{v} \mathbf{p}_{v}\left(\mathbf{x}_{t}-\mathbf{x}_{v}\right)
$$

(iv) The data set $S$ is rationalizable by an increasing, concave and continuous utility function.

The theorem shows that when budget sets are linear, GARP (statement (ii)) provides a necessary and sufficient condition for the data to be rationalizable by a concave, increasing and continuous utility function. Statement (iii) gives an equivalent condition in terms of so-called Afriat inequalities, which are linear in the unknown variables $U_{t}$ and $\lambda_{t}$. Afriat's theorem has a remarkable implication: data rationalizability by a nonsatiated utility function (statement (i)) is equivalent to rationalizability by an increasing and concave utility function (statement (iv)). As such, the theorem shows that, if budget sets are linear, it is impossible to accept rationalizability by a non-satiated utility function while rejecting it for a concave and increasing utility function. Essentially, this means that, under utility maximization, the property of concavity of utility functions is nontestable in the case of linear budget sets. 
Non-convex rationalizations on nonlinear budget sets. The picture changes drastically if budgets are nonlinear. In such a setting, GARP consistency remains necessary and sufficient for rationalizability by an increasing utility function, but it is no longer sufficient for rationalizability by a concave utility function.

Forges and Minelli (2009) showed that GARP consistency is equivalent to rationalizability by an increasing utility function. To obtain this result, they considered the gauge function $\gamma_{t}: \mathbb{R}_{+}^{n} \rightarrow \mathbb{R}^{n}$, defined by,

$$
\gamma_{t}(\mathbf{x})=\inf \left\{\lambda>0 \mid \mathbf{x} / \lambda \in B_{t}\right\} .
$$

In words, $\gamma_{t}(\mathbf{x})$ gives for the smallest number for which $\mathbf{x} / \gamma_{t}(\mathbf{x})$ belongs to the budget set. In the case of linear budget sets, we obtain that $\gamma_{t}(\mathbf{x})=\mathbf{p}_{t} \mathbf{x} / \mathbf{p}_{t} \mathbf{x}_{t} \cdot{ }^{3}$ Then, building on a result of Fostel, Scarf, and Todd (2004), Forges and Minelli proved the following theorem.

Theorem 2 (Forges and Minelli (2009)). Consider a data set $S=\left\{B_{t}, \mathbf{x}_{t}\right\}_{t \in T}$. Then, the following statements are equivalent:

(i) The data set $S$ is rationalizable by a locally non-satiated utility function.

(ii) The data set $S$ satisfies GARP.

(iii) For all $t \in T$, there exist numbers $U_{t} \geq 0$ and $\lambda_{t}>0$ such that, for all $t, v \in T$ :

$$
U_{t}-U_{v} \leq \lambda_{v}\left(\gamma_{v}\left(\mathbf{x}_{t}\right)-1\right) .
$$

(iv) There exist an increasing and continuous utility function that rationalizes the data set $S$.

There is a clear correspondence between Theorem 1 and Theorem 2. However, a notable difference is that, for nonlinear budget sets, we lose the equivalence between rationalizability by an arbitrary (non-satiated) utility function and rationalizability by a concave utility function.

This last point is illustrated in Figure 1, which presents two budget sets, given by the surfaces enclosed by $\ell_{1}, \ell_{2}, 0$ and $\ell_{1}^{\prime}, \ell_{2}^{\prime}, 0$. The chosen bundles are represented by, respectively, the points $\mathbf{x}_{1}$ and $\mathbf{x}_{2}$. It is easy to verify that this data set satisfies GARP. However, it is not rationalizable by a concave utility function, as we show by contradiction. Specifically, let us assume that the observations are rationalizable by a concave utility function. Then, because both budget sets are convex and the rationalizing utility function is concave, there must exist hyperplanes going through the chosen bundles that separate the corresponding budget sets from the upper contour sets. In Figure 1, these hyperplanes are given by the lines $r_{1}, r_{2}$ and $r_{1}^{\prime}, r_{2}^{\prime}$. By construction, the areas below these hyperplanes contain bundles that have lower utility than the chosen bundles. As such, for any rationalizing (concave) utility function $u$, it must be that $u\left(\mathbf{x}_{2}\right)<u\left(\mathbf{x}_{1}\right)$ and $u\left(\mathbf{x}_{1}\right)<u\left(\mathbf{x}_{2}\right)$, which leads to the wanted contradiction.

Thus, we conclude that, in order to characterize rationalizability by a concave utility function under nonlinear budgets, we will have to modify the GARP condition.

\footnotetext{
${ }^{3}$ In the case of linear budget sets, we have that $\mathbf{x} / \lambda \in B_{t}$ if and only if $\mathbf{p}_{t} \mathbf{x} / \lambda \leq \mathbf{p}_{t} \mathbf{x}_{t}$. This implies that $\mathbf{p}_{t} \mathbf{x} / \mathbf{p}_{t} \mathbf{x}_{t} \leq \lambda$.
} 
Figure 1: GARP and nonlinear budget sets

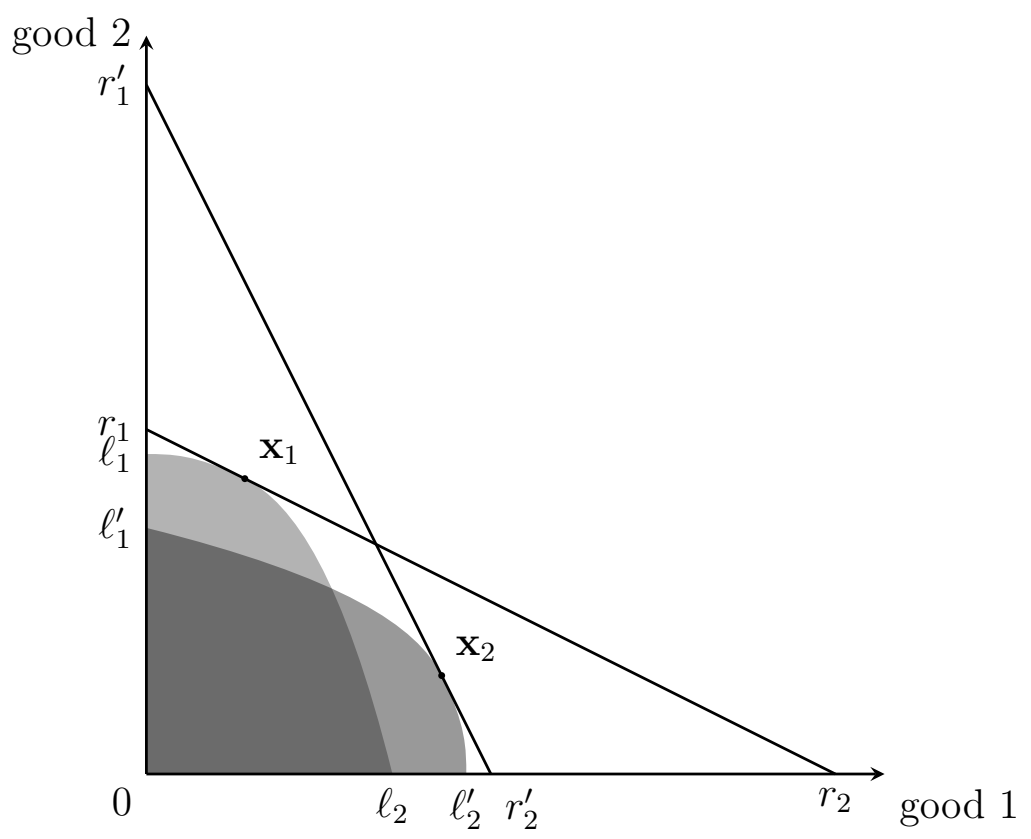

Convex rationalizations on nonlinear budget sets. In order to handle the rationalizability problem with convex preferences, we first introduce the concept of a coconvex hull. A set $H \subseteq \mathbb{R}_{+}^{n}$ is co-convex if its complement $\mathbb{R}_{+}^{n} \backslash H$ is convex. Next, take any observation $\left(B_{t}, \mathbf{x}_{t}\right)$ from a data set $S=\left\{B_{t}, \mathbf{x}_{t}\right\}_{t \in T}$. Intuitively, a co-convex hull of $\left(B_{t}, \mathbf{x}_{t}\right)$ gives a specific approximation of the set of bundles that are not better than $\mathbf{x}_{t}$. Formally, it is defined as follows.

Definition 2 (co-convex hull). Consider a data set $S=\left\{B_{t}, \mathbf{x}_{t}\right\}_{t \in T}$. The set $H_{t}$ is a co-convex hull of an observation $\left(B_{t}, \mathbf{x}_{t}\right)$ if it satisfies the following properties:

(i) $H_{t}$ is co-convex;

(ii) $H_{t}$ is closed and monotone;

(iii) $B_{t} \subseteq H_{t}$;

(iv) If $\mathbf{x} \gg \mathbf{x}_{t}$, then $\mathbf{x} \notin H_{t}$.

As explained above, the first condition requires that the complement of $H_{t}$ is convex. The second condition imposes the same conditions on $H_{t}$ as on $B_{t}$, i.e. closedness and monotonicity. The third condition requires that $H_{t}$ contains the budget set $B_{t}$, which explains the use of the term 'hull'. Finally, the fourth condition assumes that $H_{t}$ does not contain any bundle that strictly dominates $\mathbf{x}_{t}$ in all dimensions; this complies with the above interpretation of $H_{t}$ as approximating the complement of the upper contour 
set of $\mathbf{x}_{t}$. If $\mathbf{x}_{t} \in \partial B_{t}$, we have that the set $\left\{\mathbf{x} \in \mathbb{R}_{+}^{n} \mid \mathbf{x} \gg \mathbf{x}_{t}\right\}$ is a co-convex hull of $\left(\mathbf{x}_{t}, B_{t}\right)$. As such, a co-convex hull always exists if $x_{t}$ is on the boundary of $B_{t}$. The set $H_{t}$ is a minimal co-convex hull of the observation $\left(B_{t}, \mathbf{x}_{t}\right)$ if $H_{t}$ is a co-convex hull of $\left(B_{t}, \mathbf{x}_{t}\right)$ and for any other co-convex hull $H_{t}^{\prime}$ of $\left(B_{t}, \mathbf{x}_{t}\right)$, if $H_{t}^{\prime} \subseteq H_{t}$, then $H_{t}^{\prime}=H_{t}$. The existence of a minimal co-convex hull (if $x_{t}$ is on the boundary of $B_{t}$ ) can be shown by a straightforward application of Zorn's lemma. At this point, it is worth indicating that a minimal co-convex hull should not necessarily be unique.

It is well known that any closed and convex set can be written as the intersection of a collection of closed half spaces. ${ }^{4}$ As a consequence, its complement, which is an open and co-convex set, can be written as a union of open half spaces. The following exposition provides an embodiment of this idea applied to co-convex hulls.

Consider a co-convex hull $H_{t}$ of $\left(B_{t}, \mathbf{x}_{t}\right)$. By definition, the complement of this set, i.e. $\mathbb{R}_{+}^{n} \backslash H_{t}$, is an open and convex set. As such, its closure can be written as the intersection of its supporting half spaces. An element, $\mathbf{x}$, of such a half space then satisfies a condition of the form

$$
\mathbf{p}_{t}^{i} \mathbf{x} \geq m_{t}^{i}
$$

for some vector $\mathbf{p}_{t}^{i} \in \mathbb{R}^{n}$ and some number $m_{t}^{i} \in \mathbb{R}$. Consider the subset of these hyperplanes for which $\mathbf{p}_{t}^{i} \in \mathbb{R}_{+}^{n}$ and $m_{t}^{i}>0$. Any rescaling $\left(\alpha \mathbf{p}_{t}^{i}, \alpha m_{t}^{i}\right)$ of such hyperplane represents the same half space and as such we can always normalize these half spaces by setting $m_{t}^{i}=1$. Let us collect the set of these normalized vectors $\mathbf{p}_{t}^{i}$ in $A_{t}$. It is possible to show that $\mathbf{x}$ is in the closure of $\mathbb{R}_{+}^{n} \backslash H_{t}$ if and only if $\mathbf{p}_{t}^{i} \mathbf{x} \geq 1$ for all $\mathbf{p}_{t}^{i} \in A_{t}{ }^{{ }^{5}}$

The complement of the closed convex set $\mathbb{R}_{+}^{n} \backslash H_{t}$ is thus given by bundles $\mathbf{x} \in \mathbb{R}_{+}^{n}$ that satisfy $\inf _{\mathbf{p}_{t}^{i} \in A_{t}} \mathbf{p}_{t}^{i} \mathbf{x}<1$. This complement equals the interior of $H_{t}$ and therefore its closure is $H_{t}$. This implies that $H_{t}$ consists of all bundles $\mathbf{x}$ with $\inf _{\mathbf{p}_{t}^{i} \in A_{t}} \mathbf{p}_{t}^{i} \mathbf{x} \leq 1$. As a consequence, the boundary $\partial H_{t}$ can be defined by the bundles $\mathbf{x}$ for which $\inf _{\mathbf{p}_{t}^{i} \in A_{t}} \mathbf{p}_{t}^{i} \mathbf{x}=$ 1. In the following, we will slightly abuse terminology and call $A_{t}$ the support set of the co-convex hull $H_{t}$.

Our main theorem characterizes data sets for which there exists a convex rationalization, i.e. the data set is rationalizable by a quasi-concave utility function (or, equivalently, convex preferences).

Theorem 3. Consider a data set $S=\left\{B_{t}, \mathbf{x}_{t}\right\}_{t \in T}$. Then, the following statements are equivalent:

(i) The data set $S$ is rationalizable by a locally non-satiated and quasi-concave utility function.

(ii) For all $t \in T$, there exists a minimal co-convex hull $H_{t}$ of $\left(B_{t}, \mathbf{x}_{t}\right)$ such that the set $\left\{H_{t}, \mathbf{x}_{t}\right\}_{t \in T}$ satisfies GARP.

(iii) For all $t \in T$, there exists a minimal co-convex hull $H_{t}$ of $\left(B_{t}, \mathbf{x}_{t}\right)$ with associated support set $A_{t}$, and there exist numbers $U_{t} \geq 0$ and $\lambda_{t}>0$ such that, for all

\footnotetext{
${ }^{4}$ See, for example, Rockafellar (1970), Corollary 11.7.1.

${ }^{5} \mathrm{~A}$ proof of this is available upon request from the authors.
} 
$t, v \in T:$

$$
U_{t}-U_{v} \leq \lambda_{v}\left(\inf _{\mathbf{p}_{v}^{i} \in A_{v}} \mathbf{p}_{v}^{i} \mathbf{x}_{t}-1\right)
$$

(iv) The data set $S$ is rationalizable by an increasing, concave and continuous utility function.

Proof. The proof proceeds in three steps. ${ }^{6}$ First, the implication (i) $\rightarrow$ (ii) follows from the observation that, if the dataset is rationalizable by a quasi-concave utility function, then the lower contour sets of the convex indifference curve through the chosen bundle $\mathbf{x}_{t}$ contains at least one minimal co-convex hull of $\left(B_{t}, \mathbf{x}_{t}\right)$. Next, the implication (ii) $\rightarrow$ (iii) follows from Proposition 3 of Forges and Minelli (2009) by noticing that the function $\gamma_{t}(x)=\inf _{\mathbf{p}_{t}^{i} \in A_{t}} \mathbf{p}_{t}^{i} \mathbf{x}$ is in fact the gauge function of the set $H_{t} \cdot{ }^{7}$ Finally, the implication (iii) $\rightarrow$ (iv) can be obtained by considering the lower envelope function.

$$
U(\mathbf{x})=\min _{t}\left\{U_{t}+\lambda_{t}\left(\inf _{\mathbf{p}_{t}^{i} \in A_{t}} \mathbf{p}_{t}^{i} \mathbf{x}-1\right)\right\} .
$$

This function is concave (as it is the lower envelope of a collection of linear functions) and it rationalizes the data. In order to see this last property, first notice that for all $t \in T, U\left(\mathbf{x}_{t}\right)=U_{t}$. Next, assume that there is a bundle $\mathbf{x}$ in $B_{t}$ (which means that $\left.\inf _{\mathbf{p}_{t}^{i} \in A_{t}} \mathbf{p}_{t}^{i} \mathbf{x} \leq 1\right)$ and assume that $U(\mathbf{x})>U\left(\mathbf{x}_{t}\right)$. Then, we have that:

$$
U(\mathbf{x}) \leq U_{t}+\lambda_{t}\left(\inf _{\mathbf{p}_{t}^{i} \in A_{t}} \mathbf{p}_{t}^{i} \mathbf{x}-1\right) \leq U_{t}
$$

which shows the desired contradiction.

Theorem 3 clearly mirrors Afriat's theorem. The main difference pertains to statement (ii) of Theorem 3, which requires GARP consistency not in terms of the observed budget sets but in terms of a collection of minimal co-convex hulls. As indicated above, a minimal co-convex hull of $\left(B_{t}, \mathbf{x}_{t}\right)$ should not be uniquely defined in general and, therefore, verifying statement (ii) of Theorem 3 can be considerably more difficult than verifying GARP for the original data set $\left\{B_{t}, \mathbf{x}_{t}\right\}_{t \in T}$.

One final remark is in order. Statements (ii) and (iii) of Theorem 3 require the coconvex hulls $H_{t}$ to be minimal. However, one can show that the result also holds if we consider any co-convex hull rather than a minimal one. We have chosen to state Theorem 3 in its current form because the set of minimal co-convex hulls is the smallest set of co-convex hulls for which our theorem holds. This effectively makes that the theorem provides the sharpest formulation of the rationalizability conditions: in principle, it suffices to (only) consider minimal co-convex hulls when verifying rationalizability. ${ }^{8}$

\footnotetext{
${ }^{6}$ For the sake of brevity, we choose to include a compact version of the proof. The working paper version of this paper (Cherchye et al. (2012)) contains a more detailed proof.

${ }^{7}$ We thank an anonymous referee for pointing this out to us.

${ }^{8}$ Indeed, if we exclude some minimal co-convex hull to verify statement (ii) or, equivalently, statement (iii) of Theorem 3, then it may be that we erroneously reject rationalizability. Specifically, we can construct data sets that are rationalizable but violate the GARP condition for all but one minimal co-convex hull.
} 
However, by allowing any co-convex hull we can actually conclude that there exists a convex rationalization of some data set as soon as we can find one specification of coconvex hulls (not necessarily minimal) that satisfies the conditions in Theorem 3 . This will be useful for defining operational tests of rationalizability in the next section.

\section{Convex rationalizations on finite unions of poly- hedral convex sets}

The general result in Theorem 3 is essentially a theoretical one. It does not provide guidelines for testing the revealed preference conditions in specific applications. In this section, we consider the operationalization of the conditions. We show that verifying our testable implications for convex rationalizations boils down to checking linear inequalities when assuming that budget sets can be written as finite unions of polyhedral convex sets. Importantly, as indicated in the Introduction, this assumption is hardly restrictive from an applied point of view, because we can always approximate any budget set arbitrarily close as a union of polyhedral convex sets. ${ }^{9}$

Characterization. Let $B_{t}$ be a finite union of $\ell_{t}$ closed, monotonic and polyhedral convex sets $B_{t}^{j}$, each having non-empty interior. Let $K_{t}^{j}=\left\{\mathbf{q}_{t}^{j} \in \mathbf{R}_{+}^{n}\right\}$ be the set of vectors such that

$$
B_{t}^{j}=\left\{\mathbf{x} \in \mathbb{R}_{+}^{n} \mid \max _{\mathbf{q}_{t}^{j} \in K_{t}^{j}} \mathbf{q}_{t}^{j} \mathbf{x} \leq 1\right\} .
$$

For these sets, we can prove the following lemma.

Lemma 1. Consider a data set $S=\left\{B_{t}, \mathbf{x}_{t}\right\}$. If $B_{t}$ is a finite union of polyhedral convex sets and $H_{t}$ is a minimal co-convex hull of the observation $\left(B_{t}, \mathbf{x}_{t}\right)$, then

$$
H_{t}=\left\{\mathbf{x} \in \mathbb{R}_{+}^{n} \mid \min _{j=1, \ldots, \ell_{t}} \mathbf{p}_{t}^{j} \mathbf{x} \leq 1\right\}
$$

where for all $j=1, \ldots, \ell_{t}, \mathbf{p}_{t}^{j}$ is a convex combination of the vectors $\mathbf{q}_{t}^{j} \in K_{t}^{j}$ and $\min _{j=1, \ldots, \ell_{t}} \mathbf{p}_{t}^{j} \mathbf{x}_{t}=1$.

Proof. Let $H_{t}$ be a minimal co-convex hull of $\left(B_{t}, \mathbf{x}_{t}\right)$. The complement of $H_{t}$ is a convex set which is separated from all convex sets $B_{t}^{j}$. As such, for each set $B_{t}^{j}$ there exists a supporting hyperplane that separates $B_{t}^{j}$ from the complement of $H_{t}$.

We present these hyperplanes by $\left\{\mathbf{x} \in \mathbb{R}^{n} \mid \mathbf{p}_{t}^{j} \mathbf{x}=1\right\}$. From Rockafellar (1970, Theorem 17.3), we know that $\mathbf{p}_{t}^{j}=\sum_{i} \alpha_{t}^{j, i} \mathbf{q}_{t}^{j, i}$ where $\mathbf{q}_{t}^{j, i} \in K_{t}^{j}, \alpha_{t}^{j, i} \geq 0$ and $\sum_{i} \alpha_{t}^{j, i} \leq 1$. For an $\mathbf{x}$ on the intersection of this hyperplane and $B_{t}^{j}$ this gives $1=\mathbf{p}_{t}^{j} \mathbf{x}=\sum_{i} \alpha_{t}^{j, i} \mathbf{q}_{t}^{j, i} \mathbf{x}$. Using the fact that $\mathbf{q}_{t}^{j, i} \mathbf{x} \leq 1$ for all $\mathbf{q}_{t}^{i, j} \in K_{t}^{j}$, we can conclude that $\sum_{i} \alpha_{t}^{j, i}=1$.

\footnotetext{
${ }^{9}$ The operations research literature calls this inner-outer linearization. See, for example, Bertsekas (2009) for a detailed discussion.
} 
Observe that, if $\mathbf{x}_{t} \in B_{t}^{m}$, then this hyperplane must pass through $\mathbf{x}_{t}$, i.e. $\mathbf{p}_{t}^{m} \mathbf{x}_{t}=1$ while, for all other hyperplanes (characterized by $\mathbf{p}_{t}^{j}$ ), we have $\mathbf{p}_{t}^{j} \mathbf{x}_{t} \geq 1$. As such, $\min _{j=1, \ldots, \ell_{t}} \mathbf{p}_{t}^{j} \mathbf{x}_{t}=1$.

Next, define the set $H_{t}^{\prime}=\left\{\mathbf{x} \in \mathbb{R}_{+}^{n} \mid \min _{j=1, \ldots, \ell_{t}} \mathbf{p}_{t}^{j} \mathbf{x} \leq 1\right\}$. This set is co-convex (as it is the complement of the intersection of open half spaces), monotone and closed. Further, it contains each set $B_{t}^{j}$. Therefore, it also includes the budget set $B_{t}$. If $\mathbf{y} \gg \mathbf{x}_{t}$, then $\mathbf{y} \notin H_{t}^{\prime}$. Otherwise, there is a $\mathbf{p}_{t}^{j}$ such that $\left\{\mathbf{x} \in \mathbb{R}_{+}^{n} \mid \mathbf{p}_{t}^{j} \mathbf{x}=1\right\}$ intersects the complement of $H_{t}$. We conclude that $H_{t}^{\prime}$ is a co-convex hull of $\left(B_{t}, \mathbf{x}_{t}\right)$. Finally, observe that $H_{t}^{\prime} \subset H_{t}$. Given that $H_{t}$ was minimal, we obtain that $H_{t}=H_{t}^{\prime}$.

This characterization of minimal co-convex hulls allows for an efficient operationalization of the characterization in Theorem 3 for budgets sets that are finite unions of polyhedral convex budget sets. To see this, consider a collection of sets $\mathcal{H}_{t}$ such that, for all $H_{t} \in \mathcal{H}_{t}$, there exist numbers $\alpha_{t}^{j, i} \in \mathbb{R}_{+}$, with $\sum_{i=1}^{\left|K_{t}^{j}\right|} \alpha_{t}^{j, i}=1, \min _{j} \sum_{i=1}^{\left|K_{t}^{j}\right|} \alpha_{t}^{j, i} \mathbf{q}_{t}^{j, i} \mathbf{x}_{t}=1$, and

$$
H_{t}=\left\{\mathbf{x} \in \mathbb{R}_{+}^{n} \mid \min _{j=1, \ldots, \ell_{t}} \sum_{i=1}^{\left|K_{t}^{j}\right|} \alpha_{t}^{j, i} \mathbf{q}_{t}^{j, i} \mathbf{x} \leq 1\right\} .
$$

Every set in the collection $\mathcal{H}_{t}$ is clearly a co-convex hull of $\left(B_{t}, \mathbf{x}_{t}\right)$. Moreover, from Lemma 1 above we know that $\mathcal{H}_{t}$ contains all minimal co-convex hulls of $\left(B_{t}, \mathbf{x}_{t}\right)$. Thus, applying Theorem 3 to this setting gives the following result.

Proposition 1. Consider a data set $S=\left\{B_{t}, \mathbf{x}_{t}\right\}_{t \in T}$ where each $B_{t}$ is a finite union of closed, monotone polyhedral sets $B_{t}^{j}$, with $j=1, \ldots, \ell_{t}$, each having a non-empty interior. Then, the following statements are equivalent:

(i) The data set $S$ is rationalizable by a locally non-satiated and quasi-concave utility function.

(ii) For all $t \in T$ and $j=1, \ldots, \ell_{t}$, there exist numbers $\alpha_{t}^{j, i} \geq 0$, with $i=1, \ldots,\left|K_{t}^{j}\right|$, such that

- $\sum_{i=1}^{\left|K_{t}^{j}\right|} \alpha_{t}^{j, i}=1$;

- $\min _{j} \sum_{i=1}^{\left|K_{t}^{j}\right|} \alpha_{t}^{j, i} \mathbf{q}_{t}^{j, i} \mathbf{x}_{t}=1$;

- $\left\{H_{t}, \mathbf{x}_{t}\right\}_{t \in T}$ satisfies GARP, where,

$$
H_{t}=\left\{\mathbf{x} \in \mathbb{R}_{+}^{n} \mid \min _{j=1, \ldots, \ell_{t}} \sum_{i=1}^{\left|K_{t}^{j}\right|} \alpha_{t}^{j, i} \mathbf{q}_{t}^{j, i} \mathbf{x} \leq 1\right\} .
$$

(iii) For all $t \in T$ and $j=1, \ldots, \ell_{t}$, there exist numbers $U_{t} \geq 0$ and $\lambda_{t}>0$ and $\alpha_{t}^{j, i} \geq 0$, $i=1, \ldots,\left|K_{t}^{j}\right|$, such that, for all $t, v \in T$ and $k=1, \ldots \ell_{v}$ :

- $U_{t}-U_{v} \leq \lambda_{v}\left(\sum_{i=1}^{\left|K_{v}^{k}\right|} \alpha_{v}^{k, i} \mathbf{q}_{v}^{k, i} \mathbf{x}_{t}-1\right)$; 
- $\sum_{i=1}^{\left|K_{t}^{j}\right|} \alpha_{t}^{j, i}=1$;

- $\sum_{i=1}^{\left|K_{t}^{j}\right|} \alpha_{t}^{j, i} \mathbf{q}_{t}^{j, i} \mathbf{x}_{t} \geq 1$

(iv) The data set $S$ is rationalizable by an increasing, concave and continuous utility function.

Statement (iii) in this result deserves some further explanation. First of all, observe that the Afriat inequalities should hold for all $k=1, \ldots, \ell_{v}$. In this sense, the first condition is equivalent to the requirement

$$
U_{t}-U_{v} \leq \min _{k=1, \ldots, \ell_{v}} \lambda_{v}\left(\sum_{i=1}^{\left|K_{v}^{k}\right|} \alpha_{v}^{k, i} \mathbf{q}_{v}^{k, i} \mathbf{x}_{t}-1\right)=\lambda_{v}\left(\min _{k=1, \ldots, \ell_{v}} \sum_{i=1}^{\left|K_{v}^{k}\right|} \alpha_{v}^{k, i} \mathbf{q}_{v}^{k, i} \mathbf{x}_{t}-1\right)
$$

Next remark that, for $\mathbf{x}_{t} \in B_{t}^{m}$, we have $\mathbf{q}^{m, i} \mathbf{x}_{t} \leq 1$ for all $\mathbf{q}^{m, i} \in K_{t}^{m}$. As such,

$$
\sum_{i=1}^{\left|K_{t}^{m}\right|} \alpha_{t}^{m, i} \mathbf{q}_{t}^{m, i} \mathbf{x}_{t} \leq \sum_{i} \alpha_{t}^{m, i}=1
$$

Together with the third condition in statement (iii), this effectively implies

$$
\min _{j} \sum_{i=1}^{\left|K_{t}^{j}\right|} \alpha_{t}^{j, i} \mathbf{q}_{t}^{j, i} \mathbf{x}_{t}=1
$$

Operationalization. Statement (iii) in Proposition 1 is particularly convenient from a practical point of view. Specifically, it can be rewritten as requiring feasibility of the following system of linear inequalities:

For all $t \in T$ and $j=1, \ldots, \ell_{t}$, there exist numbers $U_{t} \geq 0$ and $\lambda_{t}>0$ and $\tilde{\alpha}_{t}^{j, i} \geq 0$, $i=1, \ldots,|K|_{t}^{j}$, such that, for all $t, v \in T$ and $k=1, \ldots \ell_{v}$ :

- $U_{t}-U_{v} \leq \sum_{i=1}^{\left|K_{v}^{k}\right|} \tilde{\alpha}_{v}^{k, i} \mathbf{q}_{v}^{k, i} \mathbf{x}_{t}-\lambda_{v}$

- $\sum_{i=1}^{\left|K_{t}^{j}\right|} \tilde{\alpha}_{t}^{j, i}=\lambda_{t}$

- $\sum_{i=1}^{\left|K_{t}^{j}\right|} \tilde{\alpha}_{t}^{j, i} \mathbf{q}_{t}^{j, i} \mathbf{x}_{t} \geq \lambda_{t}$.

At this point, it is worth comparing this result to an original one of Yatchew (1985). This author also considers the setting where budget sets are defined as finite unions of polyhedral convex sets. However, his analysis differs from ours in three substantial ways. First of all, he assumes that utility functions are concave. By contrast, we relax this assumption by focusing on quasi-concavity. Although this may seem like a small difference, the additional assumption of concavity (beyond quasi-concavity) greatly 
simplifies the revealed preference analysis (see, for example, Diewert (2012) for an indepth discussion of this). Second, Yatchew only considers revealed preference conditions in terms of Afriat-type inequalities (i.e. statement (iii) of Proposition 1), while we also provide conditions in terms of GARP-type restrictions (i.e. statement (ii) of Proposition 1). Finally, and most importantly, Yatchew obtains revealed preference restrictions that are quadratic in unknowns. While quadratic restrictions are in general very hard to solve, our set of inequalities can be implemented efficiently through linear programming techniques.

Labor supply application. To end this section, we consider the practical example of a labor supply setting. To obtain a realistic problem setting, the numbers that we use below are based on the Belgian tax and social security system for a single individual. ${ }^{10}$ To facilitate our discussion, we focus on a simple version of the system, and assume that individuals make labor supply decisions by composing optimal bundles of leisure and aggregate consumption. ${ }^{11}$ However, this simple set-up clearly demonstrates the kind of conclusions that can be obtained on the basis of our theoretical results.

The left panel of Figure 2 shows the budget curve (with leisure on the horizontal axis and consumption on the vertical axis) for an individual with full time gross wage (i.e. zero leisure) equal to $€ 4000$ per month. The gross wage decreases proportionally with the amount of leisure that is consumed. In practice, the net disposable income (which defines the individual's actual budget set) differs from the gross wage due to social contributions and a piecewise linear tax system. In Belgium, the social contributions amount to $6.04 \%$ of the gross income, and the piecewise linear tax system is characterized by five tax brackets (i.e. 25\%, 30\%, 40\%, 45\%, 50\%). Finally, and importantly, we need to account for a guaranteed minimum income, which equals $€ 817.26$ in the Belgian system.

Figure 2 shows that the resulting budget set is non-convex, in essence because net disposable income defined by the lowest two tax brackets is entirely below the guaranteed minimum income. Interestingly, however, this non-convex budget set can be represented as a union of two polyhedral convex sets, which makes that our theoretical results apply. More generally, it is easy to verify that any budget set defined by a minimum income level and a finite set of tax brackets (with linear tax rates for different brackets) can be written as a finite union of convex polyhedral sets. This directly motivates the empirical relevance of our theoretical contribution for labor supply analysis.

For a given setting, the testable implications that we outlined above can then be used to verify the rationality of individual labor supply decisions under different budget conditions. Alternatively, and perhaps even more interestingly, we can use our results to predict or bound labor supply responses under tax reforms (generating counterfactual changes of the tax system). We illustrate this last application in the right panel of Figure 2, which zooms in on a kink of the budget curve in the left panel. The nonlinear bold budget curve represents the pre-reform situation. Now assume a tax reform such that

\footnotetext{
${ }^{10} \mathrm{We}$ obtained our numbers from the website www.flemosi.be/easycms/MOTYFF.

${ }^{11}$ See Manski (2013) for a closely similar example.
} 
Figure 2: Labor supply setting

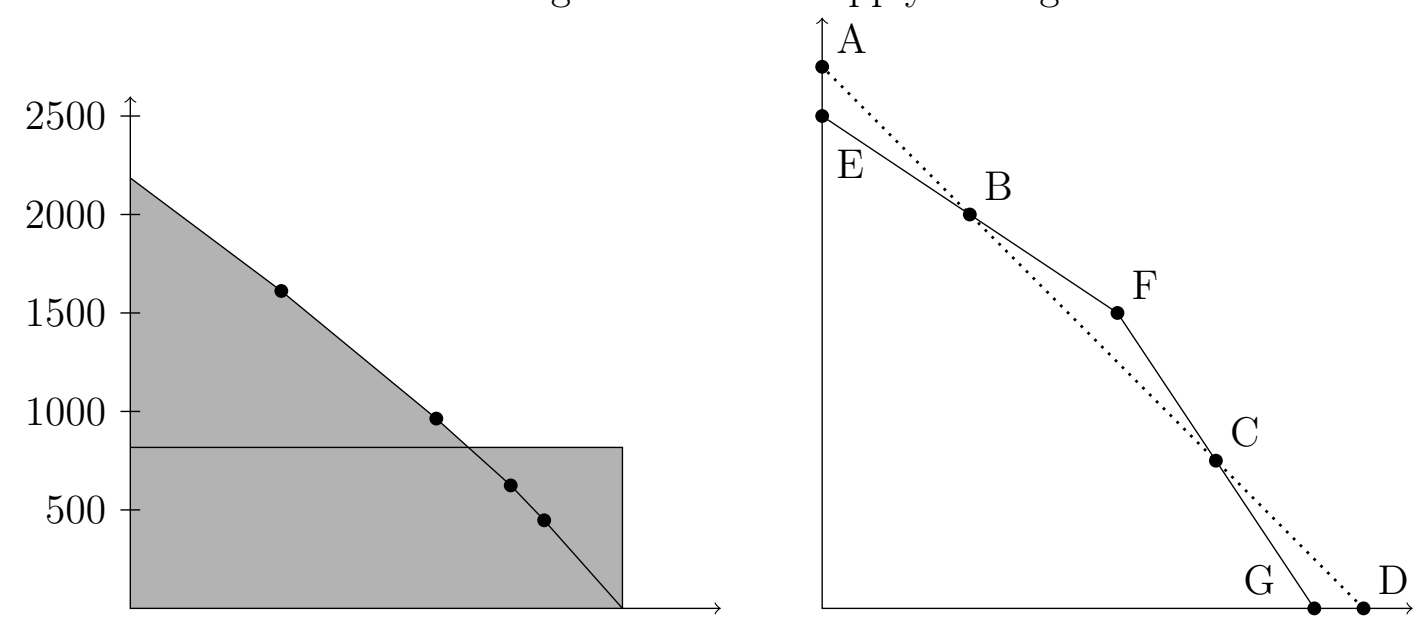

the linear dotted budget line becomes the relevant one in the post-reform period. It is then easy to show that rationality under convex preferences puts substantial structure on the post-reform leisure-consumption choices.

To see this last point, suppose that the pre-reform leisure-income bundle is situated on the line segment $E B$. Then, rationality under possibly non-convex preferences (as in Forges and Minelli (2009); see Theorem 2) only excludes post-reform bundles on the segment $B C$. Remarkably, we get exactly the same exclusion result if the pre-reform bundle is situated on the segment $C G$. In other words, if we only exploit rationality but not convexity of preferences, the specific knowledge that the initial choice is situated on $E B$ or $C G$ rules out post-reform choices on the segment $B C$. We get considerably stronger conclusions if we additionally exploit convexity of preferences (as in Proposition 1). Then, the knowledge of the initial segment (i.e. $E B$ or $C G$ ) does have significant implications. In particular, a pre-reform choice on $E B$ excludes a post-reform laborincome bundle on the entire line segment $B C D$, while a pre-reform bundle on $C G$ rules out a post-reform bundle on the segment $A B C$. Our above characterizations of convex rationalizations on nonlinear budget sets allow us to operationalize these strong implications of convex preferences in an efficient way (i.e. through linear programming). In our opinion, this convincingly shows their usefulness for practical applications.

\section{Conclusion}

We have generalized Afriat's theorem by providing a revealed preference characterization for convex rationalizations on nonlinear budget sets. This establishes the testable implications associated with rationalizing consumption behavior by a quasi-concave utility function (i.e. convex preferences). Interestingly, we also showed that, in practice, the conditions for convex rationalizations are efficiently verifiable in that they only require checking a finite number of inequalities that are linear in unknowns.

As also illustrated at the end of Section 3, our theoretical results have multiple 
possible applications. Our characterizations can be used to verify rationalizability of consumption behavior in the case of nonlinear budget sets. As indicated in the Introduction, prime examples concern labor supply, intertemporal consumption, models of household production and specific game theoretic situations. In this respect, our characterization of convex rationalizations not only allows for simply testing rationalizability. It also forms a useful basis for addressing recovery and forecasting questions under the maintained assumption of quasi-concave utility. See, for example, Varian (1982) for a detailed discussion on recovery and forecasting analysis based on Afriat's theorem (for linear budget sets). His analysis is readily translated to our setting.

Finally, our results also allow one to verify if utility functions can be assumed to be quasi-concave. This analysis can proceed in two steps. First, verify GARP consistency for the data set $\left\{B_{t}, \mathbf{x}_{t}\right\}_{t \in T}$. Subsequently, additionally check whether this data set satisfies the revealed preference conditions in Proposition 1. If the data pass the first test but fail the second, then they are rationalizable by an increasing utility function (see Theorem 2) but not by a quasi-concave one. This test can be particularly useful to check the empirical validity of economic models that heavily rely on convex preferences.

\section{References}

Afriat, S. N., 1967. The construction of utility functions from expenditure data. International Economic Review 8, 67-77.

Bertsekas, D. P., 2009. Convex Optimization Theory. Athena Scientific.

Cherchye, L., Demuynck, T., De Rock, B., 2012. Revealed preference analysis for convex rationalizations on nonlinear budget sets. Tech. rep., KULeuven.

Deaton, A., Muellbauer, J., 1980. Economics and Consumer Behavior. Cambridge University Press.

Diewert, E., 2012. Afriat's theorem and some extensions to choice under uncertainty. Economic Journal 122, 305-331.

Forges, F., Minelli, E., 2009. Afriat's theorem for general budget sets. Journal of Economic Theory 144, 135-145.

Fostel, A., Scarf, H. E., Todd, M. J., 2004. Two new proofs of Afriat's theorem. Economic Theory 24, 211-219.

Manski, C., 2013. Identification of income-leisure preferences and evaluation of income tax policy. Quantitative Economics forthcoming.

Matzkin, R. L., 1991. Axioms of revealed preference for nonlinear choice sets. Econometrica 59, 1779-1786. 
Rockafellar, T. R., 1970. Convex analysis. Princeton University Press, Chicheser, West Sussex.

Varian, H., 1982. The nonparametric approach to demand analysis. Econometrica 50, 945-974.

Yatchew, A. J., 1985. A note on nonparametric tests of consumer behavior. Economics Letters 18, 45-48. 